\title{
Le calcul fonctionnel sous-linéaire dans les espaces de Besov homogènes
}

\section{Gérard Bourdaud et Yves Meyer}

\begin{abstract}
Résumé
On établit l'estimation sous-linéaire $\|f \circ g\| \leq c(f)\|g\|$, la norme étant celle de l'espace de Besov homogène $\dot{B}_{p}^{s, q}\left(\mathbb{R}^{n}\right)$, où $1 \leq p<+\infty$, $1 \leq q \leq+\infty$ et $0<s<1+(1 / p)$. La fonction $f$ est supposée appartenir à une classe $U_{p}^{1}$ introduite antérieurement par Bourdaud et Kateb, qui contient notamment les primitives des fonctions à variation bornée sur $\mathbb{R}$. À titre d'application, on montre qu'une fonction étagée appartient à $\dot{B}_{1}^{1, \infty}\left(\mathbb{R}^{2}\right)$ si et seulement si elle appartient à $B V\left(\mathbb{R}^{2}\right)$.
\end{abstract}

\section{Introduction}

Depuis une quinzaine d'années, divers travaux ont été consacrés au calcul fonctionnel dans les espaces de Sobolev fractionnaires $W_{p}^{s}\left(\mathbb{R}^{n}\right)$, quand l'ordre de régularité vérifie $0<s<1+(1 / p)$. Ces espaces partagent la propriété d'admettre un calcul fonctionnel sous-linéaire, au sens où il existe des fonctions non affines $f: \mathbb{R} \rightarrow \mathbb{R}$ telles que l'on ait, pour une certaine constante $c$ et toute fonction $g \in W_{p}^{s}$,

$$
\|f \circ g\| \leq c\|g\|,
$$

la norme étant celle de $W_{p}^{s}$. En revanche, dès qu'on a $s>1+(1 / p)$, la condition (1.1) entraîne que $f$ est une fonction affine (cf. [13, prop. 1]). La propriété (1.1) est satisfaite par une classe naturelle de fonctions $f$, que nous définissons maintenant.

Définition 1 On appelle $B H(\mathbb{R})$ l'ensemble des fonctions continues sur la droite réelle dont la dérivée seconde, au sens des distributions, est une mesure bornée.

2000 Mathematics Subject Classification : 46E35, 47H30.

Keywords : Homogeneous Besov spaces, Composition operators, Bounded variation. 
Nous reprenons ici la notation de Savaré et Tomarelli [25], bien qu'ils l'utilisent dans un sens plus restrictif, en supposant en outre que $f$ et $f^{\prime}$ sont intégrables. Elle signifie : "à hessienne bornée", car elle est utilisée aussi bien en dimension supérieure.

Théorème 1 Soient $1 \leq p \leq \infty$ et $0<s<1+(1 / p)$. Toute fonction $f \in B H(\mathbb{R})$, telle que $f(0)=0$, opère sur $W_{p}^{s}\left(\mathbb{R}^{n}\right)$ par composition à gauche; de plus il existe une constante $c=c(s, p, n)>0$ telle que

$$
\|f \circ g\|_{W_{p}^{s}\left(\mathbb{R}^{n}\right)} \leq c\|f\|_{B H}\|g\|_{W_{p}^{s}\left(\mathbb{R}^{n}\right)} \quad, \forall g \in W_{p}^{s}\left(\mathbb{R}^{n}\right) .
$$

On verra dans la section 2.5 comment il convient de définir la seminorme $\|f\|_{B H}$. Ajoutons que, dans l'énoncé du théorème, $W_{p}^{s}\left(\mathbb{R}^{n}\right)$ désigne n'importe lequel des espaces de Sobolev d'ordre $s$ en norme $L^{p}$; autrement dit : $W_{p}^{s}\left(\mathbb{R}^{n}\right)$ est indifféremment l'espace de Besov $B_{p}^{s, q}\left(\mathbb{R}^{n}\right)$ ou l'espace de Lizorkin-Triebel $F_{p}^{s, q}\left(\mathbb{R}^{n}\right)$, pour $1 \leq q \leq \infty$. Nous renvoyons le lecteur aux articles $[6,7,9,10,14,20]$, ainsi qu'au livre de Runst et Sickel [23], pour la preuve du théorème 1 .

L'appartenance à $B H(\mathbb{R})$ n'est pas nécessaire pour que $f$ opère sur $W_{p}^{s}$. Dans le cas $0<s<1$, il est bien connu que la condition de Lipschitz est suffisante - et en un sens nécessaire, cf. [4] — pour qu'il en soit ainsi. La condition optimale, dans la plage $1 \leq s<1+(1 / p)$, est un problème ouvert. On connait cependant une classe un peu plus grande que $B H(\mathbb{R})$, dépendant de $p$, pour laquelle le théorème 1 reste vrai.

Définition 2 Pour $p \in] 1,+\infty\left[\right.$, on désigne par $\mathcal{U}_{p}(\mathbb{R})$ l'ensemble des fonctions boréliennes $f: \mathbb{R} \rightarrow \mathbb{R}$ telles que

$$
\|f\|_{\mathcal{U}_{p}}^{p}:=\sup _{t>0} \frac{1}{t} \int_{\mathbb{R}} \sup _{|h| \leq t}|f(x+h)-f(x)|^{p} d x<+\infty .
$$

On dit qu'une fonction continue $f$ appartient à $U_{p}^{1}(\mathbb{R})$ s'il existe une fonction borélienne bornée $h \in \mathcal{U}_{p}(\mathbb{R})$ telle que

$$
f(x)-f(0)=\int_{0}^{x} h(t) d t \quad, \forall x \in \mathbb{R} .
$$

On munit $U_{p}^{1}(\mathbb{R})$ de la semi-norme

$$
\|f\|_{U_{p}^{1}}:=\inf \left\{\sup _{\mathbb{R}}|h|+\|h\|_{\mathcal{U}_{p}}\right\}
$$

la borne inférieure étant étendue aux fonctions $h$ qui vérifient (1.3). 
Peut-on définir $U_{p}^{1}$ dans les cas limites $p=1$ et $p=+\infty$ ? Il est bien connu que la condition

$$
\sup _{h \neq 0} \frac{1}{|h|} \int_{\mathbb{R}}|f(x+h)-f(x)| d x<+\infty
$$

est équivalente au fait que $f^{\prime}$ est une mesure bornée. Dès lors il est naturel de poser $U_{1}^{1}(\mathbb{R}):=B H(\mathbb{R})$. Quant à $U_{\infty}^{1}(\mathbb{R})$, c'est simplement l'ensemble des fonctions lipschitziennes (cf. le paragraphe 3.3).

Dans le théorème 1 , il est possible de remplacer $B H(\mathbb{R})$ par $U_{p}^{1}(\mathbb{R})$, à condition toutefois de se limiter au cas où $W_{p}^{s}$ est un espace de Besov (l'extension aux espaces de Lizorkin-Triebel est une question ouverte). La preuve originale de ce dernier résultat, par Djalil Kateb et le premier auteur [11], est particulièrement délicate. Tout s'y passe comme si on faisait de l'interpolation réelle, à la Lions-Peetre, sans pour autant disposer d'un espace limite d'ordre $1+(1 / p)$. En fait on savait bien qu'aucun espace de Besov ou de Lizorkin-Triebel d'ordre $1+(1 / p)$ ne pouvait jouer ce rôle (cf. par exemple [13, prop. 2 et 3$])$. En revanche il était tout aussi clair qu'une inégalité du type

$$
\|f \circ g\|_{B_{p}^{1+(1 / p), \infty}\left(\mathbb{R}^{n}\right)} \leq c(f)\|g\|_{B_{p}^{1+(1 / p), 1}\left(\mathbb{R}^{n}\right)}
$$

redonnerait "gratuitement", par interpolation non linéaire, l'estimation

$$
\|f \circ g\|_{B_{p}^{s, q}\left(\mathbb{R}^{n}\right)} \leq c(f)\|g\|_{B_{p}^{s, q}\left(\mathbb{R}^{n}\right)}
$$

dans la plage $0<s<1+(1 / p)$. Savaré et Tomarelli [24, 25] furent les premiers à exploiter cette idée, en établissant l'estimation (1.4), sous la condition $f \in B H(\mathbb{R})$, redonnant ainsi une preuve élégante du théorème 1 pour les espaces de Besov. Plus récemment, M. Lanza de Cristoforis, W. Sickel et le premier auteur [13, th. 11] ont démontré l'inégalité (1.4) sous la condition $f \in U_{p}^{1}(\mathbb{R})$, simplifiant ainsi considérablement la preuve du théorème de Kateb et Bourdaud [11].

Nous nous proposons d'étendre le théorème 1, dans sa version précitée [13], aux espaces de Besov homogènes $\dot{B}_{p}^{s, q}\left(\mathbb{R}^{n}\right)$. Pourquoi une telle démarche? En premier lieu, il s'agit d'améliorer ce théorème. Pour $s>0$, l'espace de Besov usuel $B_{p}^{s, q}\left(\mathbb{R}^{n}\right)$ est en effet un sous-espace strict de $\dot{B}_{p}^{s, q}\left(\mathbb{R}^{n}\right)$, puisque

$$
B_{p}^{s, q}\left(\mathbb{R}^{n}\right)=\dot{B}_{p}^{s, q}\left(\mathbb{R}^{n}\right) \cap L^{p}\left(\mathbb{R}^{n}\right) .
$$

Ensuite il se trouve que les espaces homogènes sont préférés à leurs homologues usuels dans nombre d'applications des espaces de Besov, qu'on pense 
aux recherches sur l'équation de Navier-Stokes ou en traitement de l'image (cf. notamment Lemarié-Rieusset [18] et Meyer [19]). Enfin la preuve même du théorème 1 - y compris dans sa version originale [14] - repose sur des estimations homogènes, qu'il nous semble important d'identifier comme telles ; ainsi, dans le cas de la fonction $f(t):=|t|$ et de l'espace $B_{p}^{s, p}(\mathbb{R})$, l'estimation (1.2) est fondée sur l'inégalité

$$
\iint_{\mathbb{R}^{2}} \frac{\left.|| f\right|^{\prime}(x)-\left.|f|^{\prime}(y)\right|^{p}}{|x-y|^{s p+1}} d x d y \leq c \iint_{\mathbb{R}^{2}} \frac{\left|f^{\prime}(x)-f^{\prime}(y)\right|^{p}}{|x-y|^{s p+1}} d x d y,
$$

On peut dès lors conjecturer que, sous des hypothèses telles que $f \in$ $U_{p}^{1}(\mathbb{R})$ et $0<s<1+(1 / p)$, l'opérateur de composition $T_{f}: g \mapsto f \circ g$ envoie l'espace homogène $\dot{B}_{p}^{s, q}\left(\mathbb{R}^{n}\right)$ dans lui-même et qu'il vérifie l'estimation souslinéaire

$$
\|f \circ g\|_{\dot{B}_{p}^{s, q}\left(\mathbb{R}^{n}\right)} \leq c(f)\|g\|_{\dot{B}_{p}^{s, q}\left(\mathbb{R}^{n}\right)} .
$$

Cette idée raisonnable se heurte à diverses difficultés. Dès que $s \geq 1$, $\dot{B}_{p}^{s, q}\left(\mathbb{R}^{n}\right)$ est un espace de fonctions modulo les fonctions affines. En prenant $g(x):=x_{1}$, on voit que le second membre de (1.6) est nul, alors que le premier n'a aucune raison de l'être. Pour écarter ce premier obstacle, il suffit de réaliser $\dot{B}_{p}^{s, q}\left(\mathbb{R}^{n}\right)$ modulo les constantes, suivant une technique mise en évidence par l'un des auteurs [3], ce qui revient en quelque sorte à "tuer" les fonctions affines non constantes. Un problème subsiste cependant : l'opérateur de composition $T_{f}$ n'étant pas défini de manière univoque sur nos espaces de fonctions aux constantes près, les théorèmes classiques d'interpolation non linéaire de Peetre [21] ne peuvent s'appliquer brutalement. Nous verrons alors qu'un simple argument d'échelle permet de passer aisément de l'estimation (1.5) à son homologue homogène (1.6).

\section{Plan}

Après une section consacrée à des rappels sur les espaces de Besov homogènes et sur les fonctions à variation bornée, nous énoncerons et prouverons le théorème sur le calcul fonctionnel sous-linéaire. Dans la section 4, ce théorème sera appliqué à la comparaison des normes des fonctions étagées dans les espaces $\dot{B}_{1}^{1, \infty}\left(\mathbb{R}^{2}\right)$ et $B V\left(\mathbb{R}^{2}\right)$, un problème qui se pose dans un modèle mathématique du traitement de l'image.

\section{Notations}

On note $\mathcal{P}_{\infty}\left(\mathbb{R}^{n}\right)$ le sous-espace de $\mathcal{S}^{\prime}\left(\mathbb{R}^{n}\right)$ constitué des polynômes et par $\mathcal{P}_{m}\left(\mathbb{R}^{n}\right)$ l'ensemble des polynômes de degré au plus $m$, pour un entier $m \geq-1$ (de sorte que $\left.\mathcal{P}_{-1}\left(\mathbb{R}^{n}\right)=\{0\}\right)$. Nous noterons $[f]$ la classe 
d'équivalence d'une distribution $f \in \mathcal{S}^{\prime}\left(\mathbb{R}^{n}\right)$ modulo $\mathcal{P}_{\infty}\left(\mathbb{R}^{n}\right)$. Le symbole $\tau_{a} f$ représente la fonction translatée $x \mapsto f(x-a)$. On note $\mathbf{1}_{E}$ la fonction indicatrice (ou fonction caractéristique) d'un ensemble $E \subset \mathbb{R}^{n}$. Les lettres $c, c_{1}, \ldots$ désignent des constantes strictement positives dépendant exclusivement des paramètres $n, s, p, q$, et des fonctions auxiliaires $\psi, \gamma$, et dont la valeur peut changer d'une occurrence à l'autre. Toutes les fonctions considérées dans l'article sont supposées à valeurs réelles.

\section{Les espaces de Besov homogènes}

\subsection{Définition et premières propriétés}

Soit $\psi$ une fonction indéfiniment différentiable, paire et positive, dont le support soit un compact de $\mathbb{R}^{n} \backslash\{0\}$, et telle que

$$
\sum_{j \in \mathbb{Z}} \psi\left(2^{j} \xi\right)=1 \quad, \quad \text { pour } \quad \xi \neq 0 .
$$

Pour tout $j \in \mathbb{Z}$, l'opérateur $Q_{j}: \mathcal{S}^{\prime}\left(\mathbb{R}^{n}\right) \rightarrow \mathcal{S}^{\prime}\left(\mathbb{R}^{n}\right)$ est défini par l'identité

$$
\widehat{Q_{j} f}(\xi):=\psi\left(2^{-j} \xi\right) \widehat{f}(\xi),
$$

où $\widehat{f}$ désigne la transformée de Fourier de $f$. Notons que $Q_{j} f=0(\forall j \in \mathbb{Z})$ si et seulement si $f$ est un polynôme, et qu'on a

$$
f=\sum_{j \in \mathbb{Z}} Q_{j} f,
$$

dans l'espace quotient $\mathcal{S}^{\prime}\left(\mathbb{R}^{n}\right) / \mathcal{P}_{\infty}\left(\mathbb{R}^{n}\right)$.

Définition 3 Soient $s \in \mathbb{R}, p, q \in[1,+\infty]$. L'espace de Besov homogène $\dot{B}_{p}^{s, q}\left(\mathbb{R}^{n}\right)$ est l'ensemble des $f \in \mathcal{S}^{\prime}\left(\mathbb{R}^{n}\right) / \mathcal{P}_{\infty}\left(\mathbb{R}^{n}\right)$ tels que

$$
\|f\|_{\dot{B}_{p}^{s, q}\left(\mathbb{R}^{n}\right)}:=\left(\sum_{j \in \mathbb{Z}}\left(2^{j s}\left\|Q_{j} f\right\|_{p}\right)^{q}\right)^{1 / q}<+\infty .
$$

On montre que $\dot{B}_{p}^{s, q}\left(\mathbb{R}^{n}\right)$ est un espace de Banach pour la norme ci-dessus. L'espace de Besov homogène est ainsi dénommé car il possède de remarquables propriétés d'invariance sous l'action du groupe des similitudes. On a plus précisément :

$$
\begin{gathered}
\left\|\tau_{a} f\right\|_{\dot{B}_{p}^{s, q}\left(\mathbb{R}^{n}\right)}=\|f\|_{\dot{B}_{p}^{s, q}\left(\mathbb{R}^{n}\right)} \quad, \quad \forall a \in \mathbb{R}^{n} . \\
c_{1}\|f\|_{\dot{B}_{p}^{s, q}\left(\mathbb{R}^{n}\right)} \leq \lambda^{(n / p)-s}\|f(\lambda(\cdot))\|_{\dot{B}_{p}^{s, q}\left(\mathbb{R}^{n}\right)} \leq c_{2}\|f\|_{\dot{B}_{p}^{s, q}\left(\mathbb{R}^{n}\right)} \quad, \forall \lambda>0 .
\end{gathered}
$$


On peut d'ailleurs remplacer $\|-\|_{\dot{B}_{p}^{s, q}\left(\mathbb{R}^{n}\right)}$ par une norme équivalente $\|-\|^{\prime}$ qui vérifie encore la propriété (2.3) et améliore la propriété (2.4), au sens où

$$
\lambda^{(n / p)-s}\|f(\lambda(\cdot))\|^{\prime}=\|f\|^{\prime} \quad, \forall \lambda>0 .
$$

Il suffit pour cela de remplacer la partition discrète (2.1) par une partition continue (cf. par exemple [5]).

Proposition 1 Soient $s \in \mathbb{R}, p, q \in[1,+\infty]$. L'espace de Besov $\dot{B}_{p}^{s, q}\left(\mathbb{R}^{n}\right)$ est l'ensemble des $f \in \mathcal{S}^{\prime}\left(\mathbb{R}^{n}\right) / \mathcal{P}_{\infty}\left(\mathbb{R}^{n}\right)$ tels que $\partial_{j} f \in \dot{B}_{p}^{s-1, q}\left(\mathbb{R}^{n}\right)$ pour tout $j=1, \ldots, n$. De plus l'expression suivante est une norme équivalente sur $\dot{B}_{p}^{s, q}\left(\mathbb{R}^{n}\right):$

$$
\sum_{j=1}^{n}\left\|\partial_{j} f\right\|_{\dot{B}_{p}^{s-1, q}\left(\mathbb{R}^{n}\right)} .
$$

Esquisse de la preuve. Considérons une fonction $\gamma \in \mathcal{S}\left(\mathbb{R}^{n}\right)$ telle que $\widehat{\gamma} \psi=\psi$ et posons $\gamma_{j}(x):=2^{j n} \gamma\left(2^{j} x\right)$, pour tout $j \in \mathbb{Z}$. On a alors

$$
Q_{j} f=\gamma_{j} * Q_{j} f \quad, \forall f \in \mathcal{S}^{\prime}\left(\mathbb{R}^{n}\right),
$$

et donc

$$
\left\|Q_{j}\left(\partial_{k} f\right)\right\|_{p}=\left\|\partial_{k} \gamma_{j} * Q_{j} f\right\|_{p} \leq\left\|\partial_{k} \gamma_{j}\right\|_{1}\left\|Q_{j} f\right\|_{p}=c 2^{j}\left\|Q_{j} f\right\|_{p},
$$

pour tout $k=1, \ldots, n$. Cette dernière estimation entraîne aussitôt

$$
\sum_{k=1}^{n}\left\|\partial_{k} f\right\|_{\dot{B}_{p}^{s-1, q}\left(\mathbb{R}^{n}\right)} \leq c\|f\|_{\dot{B}_{p}^{s, q}\left(\mathbb{R}^{n}\right)} .
$$

L'estimation en sens opposé est un peu plus délicate à établir (voir, par exemple, [5, prop. 8]).

En tant qu'espaces de distributions, les espaces de Besov possèdent l'importante propriété de Fatou, décrite par l'énoncé suivant.

Proposition 2 Soient $s \in \mathbb{R}, p, q \in[1,+\infty]$. Si $\left(f_{k}\right)_{k \in \mathbb{N}}$ est une suite bornée dans $\dot{B}_{p}^{s, q}\left(\mathbb{R}^{n}\right)$ et si $\lim _{k \rightarrow+\infty} f_{k}=f$ au sens des distributions tempérées, alors $f \in \dot{B}_{p}^{s, q}\left(\mathbb{R}^{n}\right)$ et

$$
\|f\|_{\dot{B}_{p}^{s, q}\left(\mathbb{R}^{n}\right)} \leq c \sup _{k \geq 0}\left\|f_{k}\right\|_{\dot{B}_{p}^{s, q}\left(\mathbb{R}^{n}\right)} .
$$

Preuve. La preuve donnée par Franke [17] pour les espaces de Besov non homogènes s'adapte sans difficulté aux espaces homogènes. 


\subsection{Les espaces de Besov non homogènes}

Définition 4 Pour $s>0$, l'espace de Besov usuel (non homogène) $B_{p}^{s, q}\left(\mathbb{R}^{n}\right.$ ) est l'ensemble des fonctions $f \in L^{p}\left(\mathbb{R}^{n}\right)$ telles que $[f] \in \dot{B}_{p}^{s, q}\left(\mathbb{R}^{n}\right)$.

On montre que $B_{p}^{s, q}\left(\mathbb{R}^{n}\right)$ est un espace de Banach de distributions tempérées pour la norme

$$
\|f\|_{B_{p}^{s, q}\left(\mathbb{R}^{n}\right)}:=\|f\|_{\dot{B}_{p}^{s, q}\left(\mathbb{R}^{n}\right)}+\|f\|_{p} .
$$

On peut introduire les espaces $B_{p}^{s, q}\left(\mathbb{R}^{n}\right)$ quel que soit le réel $s$, à l'aide d'une partition de l'unité de l'espace des fréquences analogue à (2.1) (voir, par exemple, $[22,26,27])$. Nous adoptons la définition 4 - équivalente aux définitions de Peetre et Triebel dans le cas $s>0$ - car, dans le cadre de cet article, elle nous semble la plus simple et la plus utile.

\subsection{Réalisations des espaces de Besov}

Il s'avère naturel et utile de représenter les espaces homogènes comme des sous-espaces de $\mathcal{S}^{\prime}\left(\mathbb{R}^{n}\right) / \mathcal{P}_{m}\left(\mathbb{R}^{n}\right)$, où l'entier $m$ est aussi petit que possible.

Définition 5 Une réalisation modulo $\mathcal{P}_{m}\left(\mathbb{R}^{n}\right)$ de $\dot{B}_{p}^{s, q}\left(\mathbb{R}^{n}\right)$ est une application linéaire continue

$$
\sigma: \dot{B}_{p}^{s, q}\left(\mathbb{R}^{n}\right) \rightarrow \mathcal{S}^{\prime}\left(\mathbb{R}^{n}\right) / \mathcal{P}_{m}\left(\mathbb{R}^{n}\right)
$$

telle que $[\sigma(f)]=f$, pour tout $f \in \dot{B}_{p}^{s, q}\left(\mathbb{R}^{n}\right)$.

Une telle réalisation est un isomorphisme linéaire de $\dot{B}_{p}^{s, q}\left(\mathbb{R}^{n}\right)$ sur son image, de sorte que $\sigma\left(\dot{B}_{p}^{s, q}\left(\mathbb{R}^{n}\right)\right)$ devient un espace de Banach si on le munit de la norme

$$
\|\sigma(f)\|:=\|f\|_{\dot{B}_{p}^{s, q}\left(\mathbb{R}^{n}\right)} .
$$

Par abus de langage, l'espace $\sigma\left(\dot{B}_{p}^{s, q}\left(\mathbb{R}^{n}\right)\right)$ est aussi appelé réalisation de $\dot{B}_{p}^{s, q}\left(\mathbb{R}^{n}\right)$ : il y a en effet une correspondance bijective évidente entre les réalisations et leurs images. Pour toute valeur de $m$, l'espace $\dot{B}_{p}^{s, q}\left(\mathbb{R}^{n}\right)$ admet une infinité de réalisations modulo $\mathcal{P}_{m}$. Pour certaines valeurs de $m$, il en admet une qui commute avec les translations et avec les dilatations. On la construit de la manière suivante. on pose

Si $f \in \mathcal{S}^{\prime}\left(\mathbb{R}^{n}\right) / \mathcal{P}_{\infty}\left(\mathbb{R}^{n}\right)$ et si la série $\sum_{j \in \mathbb{Z}} Q_{j} f$ converge dans $\mathcal{S}^{\prime}\left(\mathbb{R}^{n}\right) / \mathcal{P}_{m}\left(\mathbb{R}^{n}\right)$,

$$
\sigma_{m}(f):=\sum_{j \in \mathbb{Z}} Q_{j} f \in \mathcal{S}^{\prime}\left(\mathbb{R}^{n}\right) / \mathcal{P}_{m}\left(\mathbb{R}^{n}\right)
$$


Proposition 3 On définit l'entier $\mu=\mu(s, p, q, n)$ comme suit :

$$
\mu:= \begin{cases}\max \left(E\left(s-\frac{n}{p}\right),-1\right), & \text { si } q>1 \text { ou } s-\frac{n}{p} \notin \mathbb{N}, \\ s-\frac{n}{p}-1, & \text { si } q=1 \text { et } s-\frac{n}{p} \in \mathbb{N} .\end{cases}
$$

Alors $\sigma_{\mu}(f)$ est défini pour tout $f \in \dot{B}_{p}^{s, q}\left(\mathbb{R}^{n}\right)$. L'application $\sigma_{\mu}$ ainsi définie est une réalisation de $\dot{B}_{p}^{s, q}\left(\mathbb{R}^{n}\right)$ modulo $\mathcal{P}_{\mu}\left(\mathbb{R}^{n}\right)$ qui commute avec les translations et avec les dilatations.

Le lecteur trouvera une preuve de la proposition 3 dans l'article [8]. Notons que l'entier $\mu$ est minimal au sens suivant : s'il existe une réalisation

$$
\sigma: \dot{B}_{p}^{s, q}\left(\mathbb{R}^{n}\right) \rightarrow \mathcal{S}^{\prime}\left(\mathbb{R}^{n}\right) / \mathcal{P}_{m}\left(\mathbb{R}^{n}\right)
$$

qui commute aux les translations, alors $m \geq \mu$ et, pour tout $f \in \dot{B}_{p}^{s, q}\left(\mathbb{R}^{n}\right)$, $\sigma(f)$ n'est autre que la classe d'équivalence de $\sigma_{\mu}(f)$ modulo $\mathcal{P}_{m}\left(\mathbb{R}^{n}\right)$. La preuve de cette assertion se trouve pour l'essentiel dans l'article [3].

Pour $s<n / p$, il résulte de la proposition 3 que l'espace de Besov admet une réalisation canonique comme sous-espace de $\mathcal{S}^{\prime}\left(\mathbb{R}^{n}\right)$. Pour la décrire, il suffit de disposer d'une notion de distribution tendant vers 0 à l'infini, car c'est le moyen le plus sûr pour "éliminer" les polynômes.

Définition 6 On dit qu'une distribution tempérée $f \in \mathcal{S}^{\prime}\left(\mathbb{R}^{n}\right)$ tend vers 0 à l'infini si on a

$$
\lim _{\lambda \rightarrow 0} f\left(\frac{\dot{\lambda}}{\lambda}\right)=0
$$

dans $\mathcal{S}^{\prime}\left(\mathbb{R}^{n}\right)$. L'ensemble des telles distributions est noté $\widetilde{C}_{0}\left(\mathbb{R}^{n}\right)$.

Voici quelques exemples de distributions tendant vers 0 à l'infini :

- les fonctions appartenant à $C_{0}\left(\mathbb{R}^{n}\right)$ ou à $L^{p}\left(\mathbb{R}^{n}\right)$, pour $1 \leq p<+\infty$;

- les mesures boréliennes bornées;

- les dérivées des fonctions continues bornées;

- les dérivées des distributions appartenant à $\widetilde{C}_{0}\left(\mathbb{R}^{n}\right)$.

Proposition 4 Si $s<n / p$, ou si $s=n / p$ et $q=1$, alors, pour tout élément $f$ de $\dot{B}_{p}^{s, q}\left(\mathbb{R}^{n}\right)$, la distribution tempérée $\sigma_{-1}(f)$ est l'unique représentant de $f$ tendant vers zéro à l'infini.

Preuve. Voir [3, pp. 46-47], ainsi que la preuve de la proposition 5 ci-dessous.

Nous concluerons cette présentation des espaces de Besov homogènes en fixant, pour la suite de l'article, la version de $\dot{B}_{p}^{s, q}\left(\mathbb{R}^{n}\right)$ qui s'avère pertinente en vue du calcul fonctionnel sous-linéaire. 
Proposition 5 On suppose que les paramètres $s, p, q$ satisfont les conditions suivantes :

$-1 \leq p<\infty$,

- $0<s<1+(1 / p)$ et $1 \leq q \leq+\infty$, ou $s=1+(1 / p)$ et $q=1$.

Soit $\dot{\mathcal{B}}_{p}^{s, q}\left(\mathbb{R}^{n}\right)$ l'ensemble des distributions tempérées $f$ telles que

$$
[f] \in \dot{B}_{p}^{s, q}\left(\mathbb{R}^{n}\right) \quad \text { et } \quad \partial_{j} f \in \widetilde{C}_{0}\left(\mathbb{R}^{n}\right) \quad \text { pour } \quad j=1, \ldots, n .
$$

Tout élément de $\dot{B}_{p}^{s, q}\left(\mathbb{R}^{n}\right)$ admet un représentant dans $\dot{\mathcal{B}}_{p}^{s, q}\left(\mathbb{R}^{n}\right)$, unique à l'addition près d'une constante. L'espace $\dot{\mathcal{B}}_{p}^{s, q}\left(\mathbb{R}^{n}\right)$ sera muni de la seminorme $\|-\|_{\dot{B}_{p}^{s, q}\left(\mathbb{R}^{n}\right)}$.

Preuve. C'est une conséquence immédiate des propositions 1 et 4 . Cependant, il nous sera utile de connaître explicitement la réalisation

$$
\sigma: \dot{B}_{p}^{s, q}\left(\mathbb{R}^{n}\right) \rightarrow \dot{\mathcal{B}}_{p}^{s, q}\left(\mathbb{R}^{n}\right)
$$

Soit donc $g \in \dot{B}_{p}^{s, q}\left(\mathbb{R}^{n}\right)$.

Grâce à la condition $s>0$, on a

$$
\sum_{j \geq 0}\left\|Q_{j} g\right\|_{p}=\sum_{j \geq 0} 2^{-j s}\left(2^{j s}\left\|Q_{j} g\right\|_{p}\right) \leq c\|g\|_{\dot{B}_{p}^{s, q}\left(\mathbb{R}^{n}\right)},
$$

de sorte que la série $\sum_{j \geq 0} Q_{j} g$ converge normalement dans $L^{p}\left(\mathbb{R}^{n}\right)$. L'étude de la série $\sum_{j<0} Q_{j} g$ nécessite une discussion suivant la position de $s$ par rapport à $n / p$.

1 er cas. Supposons $s<n / p$ (ou $s=n / p$ et $q=1)$. À l'aide de l'identité (2.6), on obtient

$$
\sum_{j<0}\left\|Q_{j} g\right\|_{\infty} \leq c \sum_{j<0} 2^{j(n / p)}\left\|Q_{j} g\right\|_{p} \leq c \sum_{j<0} 2^{j((n / p)-s)}\left(2^{j s}\left\|Q_{j} g\right\|_{p}\right) .
$$

De plus, par la condition $p<\infty$, on a $Q_{j} g \in C_{0}\left(\mathbb{R}^{n}\right)$. Par hypothèse sur $s$, on en déduit la convergence normale de la série $\sum_{j<0} Q_{j} g$ dans $C_{0}\left(\mathbb{R}^{n}\right)$. La série $\sum_{j \in \mathbb{Z}} Q_{j} g$ est convergente dans l'espace normé $C_{0}\left(\mathbb{R}^{n}\right)+L^{p}\left(\mathbb{R}^{n}\right)$ et sa somme est une distribution tendant vers zéro à l'infini. C'est donc un représentant de $g$ appartenant à $\dot{\mathcal{B}}_{p}^{s, q}\left(\mathbb{R}^{n}\right)$.

2ième cas. Supposons $n / p \leq s<(n / p)+1$.

Il sera commode d'introduire l'ensemble $E\left(\mathbb{R}^{n}\right)$ des fonctions continues $f$ sur $\mathbb{R}^{n}$ telles que $f(x)=o(|x|)$ quand $|x| \rightarrow+\infty$, car il possède la propriété suivante. 
Lemme 1 Pour toute fonction $f \in E\left(\mathbb{R}^{n}\right)$, les dérivées premières $\partial_{j} f$, $j=1, \ldots, n$, appartiennent à $\widetilde{C}_{0}\left(\mathbb{R}^{n}\right)$.

Preuve du lemme. Pour $\varphi \in \mathcal{S}\left(\mathbb{R}^{n}\right)$, on a

$$
\left|\left\langle\partial_{j} f\left(\frac{\dot{\lambda}}{\lambda}\right), \varphi\right\rangle\right| \leq|\lambda| \int_{\mathbb{R}^{n}}\left|f\left(\frac{x}{\lambda}\right)\right|\left|\partial_{j} \varphi(x)\right| d x .
$$

Pour un $\varepsilon>0$ donné, on choisit $R>0$ tel que

$$
\sup _{|x| \geq R} \frac{|f(x)|}{|x|} \leq \varepsilon .
$$

Soit $M(R):=\max _{|x| \leq R}|f(x)|$. Il vient alors

$$
\left|\left\langle\partial_{j} f\left(\frac{\dot{\lambda}}{\lambda}\right), \varphi\right\rangle\right| \leq \varepsilon \int_{\mathbb{R}^{n}}|x|\left|\partial_{j} \varphi(x)\right| d x+|\lambda| M(R) \int_{\mathbb{R}^{n}}\left|\partial_{j} \varphi(x)\right| d x,
$$

d'où on déduit que $\lim _{\lambda \rightarrow 0}\left\langle\partial_{j} f\left(\frac{\dot{\lambda}}{\lambda}\right), \varphi\right\rangle=0$.

À l'aide de l'identité (2.6), on obtient, pour tout $x \in \mathbb{R}^{n}$ et tout entier $j<0$,

$$
\left|Q_{j} g(x)-Q_{j} g(0)\right| \leq|x|\left\|\nabla\left(Q_{j} g\right)\right\|_{\infty} \leq c\|g\|_{\dot{B}_{p}^{s, q}\left(\mathbb{R}^{n}\right)}|x| 2^{j(1+(n / p)-s)} .
$$

Il en découle que la série de fonctions $\sum_{j<0}\left(Q_{j} g-Q_{j} g(0)\right)$ converge normalement sur tout compact de $\mathbb{R}^{n}$. Sa somme est donc une fonction continue $h$. De plus en choisissant l'entier $k \geq 1$ de sorte que $2^{k} \leq|x|<2^{k+1}$, on voit que

$$
\begin{gathered}
\sum_{j<0}\left|Q_{j} g(x)-Q_{j} g(0)\right| \leq \sum_{j<-k}|x|\left\|\nabla\left(Q_{j} g\right)\right\|_{\infty}+2 \sum_{j=-k}^{-1}\left\|Q_{j} g\right\|_{\infty} \\
\leq c\|g\|_{\dot{B}_{p}^{s, q}\left(\mathbb{R}^{n}\right)}\left(|x| \sum_{j<-k} 2^{j(1+(n / p)-s)}+2 \sum_{j=-k}^{-1} 2^{j((n / p)-s)}\right),
\end{gathered}
$$

de sorte que

$$
h(x)=O\left(|x|^{s-(n / p)}\right) \quad \text { si } s>n / p, \quad \text { et } \quad h(x)=O(\log |x|) \quad \text { si } s=n / p,
$$

quand $|x|$ tend vers l'infini. La fonction

$$
h+\sum_{j \geq 0} Q_{j} g
$$

est un élément de $E\left(\mathbb{R}^{n}\right)+L^{p}\left(\mathbb{R}^{n}\right)$; d'après le lemme 1, c'est un représentant de $g$ appartenant à $\dot{\mathcal{B}}_{p}^{s, q}\left(\mathbb{R}^{n}\right)$. 
3ième cas. Supposons $s=(n / p)+1$. Compte tenu des hypothèses sur $s$, on a alors $n=q=1$. L'identité (2.6) nous donne, pour tout $x \in \mathbb{R}$ et tout $j \in \mathbb{Z}$

$$
\left|Q_{j} g(x)-Q_{j} g(0)\right| \leq c|x| 2^{j s}\left\|Q_{j} g\right\|_{p},
$$

alors que

$$
\left\|\left(Q_{j} g\right)^{\prime}\right\|_{\infty} \leq c 2^{j s}\left\|Q_{j} g\right\|_{p}
$$

Il en découle que la série de fonctions $\sum_{j \in \mathbb{Z}}\left(Q_{j} g-Q_{j} g(0)\right)$ converge normalement sur tout compact de $\mathbb{R}$ et que sa somme est une fonction de classe $C^{1}$ dont la dérivée appartient à $C_{0}(\mathbb{R})$; cette fonction est donc un représentant de $g$ appartenant à $\dot{\mathcal{B}}_{p}^{s, q}(\mathbb{R})$.

\subsection{Approximation par des fonctions de $B_{p}^{s, q}\left(\mathbb{R}^{n}\right)$}

Comme nous l'avons annoncé en introduction, notre preuve de l'estimation (1.6) consiste à extrapoler l'estimation (1.5). Pour ce faire on dispose d'une procédure standard pour approcher les fonctions de $\dot{\mathcal{B}}_{p}^{s, q}\left(\mathbb{R}^{n}\right)$ par des fonctions de $B_{p}^{s, q}\left(\mathbb{R}^{n}\right)$.

Proposition 6 Sous les hypothèses de la proposition 5, on considère une fonction $g \in \dot{\mathcal{B}}_{p}^{s, q}\left(\mathbb{R}^{n}\right)$. Il existe alors une suite $\left(g_{k}\right)_{k \geq 1}$ dans $B_{p}^{s, q}\left(\mathbb{R}^{n}\right)$ et une suite numérique $\left(a_{k}\right)_{k \geq 1}$ telles que

(i) $\left\|g_{k}\right\|_{\dot{B}_{p}^{s, q}\left(\mathbb{R}^{n}\right)} \leq c\|g\|_{\dot{B}_{p}^{s, q}\left(\mathbb{R}^{n}\right)}$;

(ii) $g_{k}+a_{k} \rightarrow g$ dans $L_{l o c}^{1}\left(\mathbb{R}^{n}\right)$.

Preuve. Il nous suffira d'exploiter à nouveau la preuve de la proposition 5 . On définit la suite $\left(g_{k}\right)_{k \geq 1}$ par

$$
g_{k}:=\sum_{j \geq-k} Q_{j} g
$$

On sait déjà que $g_{k} \in L^{p}\left(\mathbb{R}^{n}\right)$, et on voit facilement que

$$
\left\|g_{k}\right\|_{\dot{B}_{p}^{s, q}\left(\mathbb{R}^{n}\right)} \leq c\left(\sum_{j \geq-k}\left(2^{j s}\left\|Q_{j} g\right\|_{p}\right)^{q}\right)^{1 / q} \leq c\|g\|_{\dot{B}_{p}^{s, q}\left(\mathbb{R}^{n}\right)} .
$$

Pour établir la propriété (ii), on discute suivant la position de $s$ par rapport à $n / p$.

1 er cas. Supposons $s<n / p$ (ou $s=n / p$ et $q=1$ ). On sait que la série $\sum_{j \in \mathbb{Z}} Q_{j} g$ est convergente dans l'espace normé $C_{0}\left(\mathbb{R}^{n}\right)+L^{p}\left(\mathbb{R}^{n}\right)$. La suite $\left(g_{k}\right)_{k \geq 1}$ a donc une limite $h$ dans $L_{l o c}^{1}\left(\mathbb{R}^{n}\right)$, telle que $h-g$ soit une constante. 
2ième cas. Supposons $n / p \leq s<(n / p)+1$. Posons

$$
a_{k}:=-\sum_{j=-k}^{-1} Q_{j} g(0) \text {. }
$$

Puisque

$$
\sum_{j<-k}\left|Q_{j} g(x)-Q_{j} g(0)\right| \leq c\|g\|_{\dot{B}_{p}^{s, q}\left(\mathbb{R}^{n}\right)}|x| 2^{k(s-1-(n / p))},
$$

pour tout $x \in \mathbb{R}^{n}$ et tout entier $k \geq 1$, on conclut que la suite $\left(g_{k}+a_{k}\right)_{k \geq 1}$ admet une limite $h$ dans $L_{l o c}^{1}\left(\mathbb{R}^{n}\right)$, telle que $h-g$ soit une constante.

3ième cas. Supposons $s=(n / p)+1$, et donc $n=q=1$. On a

$$
\left\|Q_{j} g\right\|_{\infty} \leq c 2^{-j}\|g\|_{\dot{B}_{p}^{s, q}(\mathbb{R})},
$$

de sorte que les constantes

$$
a_{k}:=-\sum_{j \geq-k} Q_{j} g(0)
$$

sont bien définies. La fonction

$$
h:=\sum_{j \in \mathbb{Z}}\left(Q_{j} g-Q_{j} g(0)\right)
$$

est la limite de la suite $\left(g_{k}+a_{k}\right)_{k \geq 1}$ dans $L_{l o c}^{1}(\mathbb{R})$ et la fonction $h-g$ est une constante.

\subsection{Fonctions à variation bornée}

\subsubsection{Généralités}

On définit classiquement $B V\left(\mathbb{R}^{n}\right)$ comme l'ensemble des distributions sur $\mathbb{R}^{n}$ dont les dérivées premières sont des mesures boréliennes bornées. À tout $f \in B V\left(\mathbb{R}^{n}\right)$ on associe la semi-norme

$$
\nu(f):=\sum_{j=1}^{n}\left\|\partial_{j} f\right\|_{M},
$$

où $\|g\|_{M}$ désigne la variation totale de la mesure $g$. On sait que $f \in B V\left(\mathbb{R}^{n}\right)$ si et seulement si

$$
\|f\|_{B V}:=\sup _{h \in \mathbb{R}^{n} \backslash\{0\}} \frac{1}{|h|} \int_{\mathbb{R}^{n}}|f(x+h)-f(x)| d x<+\infty
$$

(voir e.g., DeVore et Lorentz [16, ch. 2, th. 9.3]) et que les semi-normes $\nu$ et $\|-\|_{B V}$ sont équivalentes. 
Par sa définition même, $B V\left(\mathbb{R}^{n}\right)$ peut être assimilé à un espace de Sobolev d'ordre 1 , en norme $L^{1}$. J. Peetre $[22$, th. 7, p. 112] a donné à cette affirmation vague le contenu précis que voici :

Proposition 7 On a

$$
\dot{\mathcal{B}}_{1}^{1,1}\left(\mathbb{R}^{n}\right) \subset B V\left(\mathbb{R}^{n}\right) \subset \dot{\mathcal{B}}_{1}^{1, \infty}\left(\mathbb{R}^{n}\right)
$$

et les deux injections canoniques sont continues.

Preuve. 1.- Soit $g \in \dot{\mathcal{B}}_{1}^{1,1}\left(\mathbb{R}^{n}\right)$ et $k=1, \ldots, n$. On a

$$
\partial_{k} g=\sum_{j \in \mathbb{Z}} Q_{j}\left(\partial_{k} g\right)
$$

dans $\mathcal{S}^{\prime}\left(\mathbb{R}^{n}\right)$. Grâce à (2.6), il vient

$$
\left\|Q_{j}\left(\partial_{k} g\right)\right\|_{1}=\left\|\partial_{k} \gamma_{j} * Q_{j} g\right\|_{1} \leq c 2^{j}\left\|Q_{j} g\right\|_{1}
$$

ce qui montre que la série $\sum_{j \in \mathbb{Z}} Q_{j}\left(\partial_{k} g\right)$ converge normalement dans $L^{1}\left(\mathbb{R}^{n}\right)$. On en déduit que $\partial_{k} g \in L^{1}\left(\mathbb{R}^{n}\right)$ et que

$$
\nu(g)=\sum_{k=1}^{n} \int_{\mathbb{R}^{n}}\left|\partial_{k} g(x)\right| d x \leq c\|g\|_{\dot{B}_{1}^{1,1}\left(\mathbb{R}^{n}\right)} .
$$

2.- Soit $g \in B V\left(\mathbb{R}^{n}\right)$. Puisque $Q_{j}\left(\partial_{k} g\right)$ n'est autre que la convolution de la mesure $\partial_{k} g$ avec la fonction $x \mapsto 2^{j n}\left(\mathcal{F}^{-1} \psi\right)\left(2^{j} x\right)$, on a

$$
\left\|Q_{j}\left(\partial_{k} g\right)\right\|_{1} \leq\left\|\mathcal{F}^{-1} \psi\right\|_{1}\left\|\partial_{k} g\right\|_{M},
$$

soit encore

$$
\left\|\partial_{k} g\right\|_{\dot{B}_{1}^{0, \infty}\left(\mathbb{R}^{n}\right)} \leq c\left\|\partial_{k} g\right\|_{M} \quad, k=1, \ldots, n .
$$

Grâce à la proposition 1 , on obtient $\|g\|_{\dot{B}_{1}^{1, \infty}\left(\mathbb{R}^{n}\right)} \leq c \nu(g)$. Puisque $\partial_{k} g$ est une mesure bornée, on a $\partial_{k} g \in \widetilde{C}_{0}\left(\mathbb{R}^{n}\right)$, pour $k=1, \ldots, n$, ce qui achève de prouver que $g \in \dot{\mathcal{B}}_{1}^{1, \infty}\left(\mathbb{R}^{n}\right)$.

\subsubsection{Le cas de la dimension 1}

On voit aisément que $B V(\mathbb{R})$ s'injecte dans $L^{\infty}(\mathbb{R})$ et que

$$
f \mapsto \nu(f)+\|f\|_{\infty}
$$

est une norme pour laquelle $B V(\mathbb{R})$ est un espace de Banach de distributions, invariant par translations. Suivant la définition $1, B H(\mathbb{R})$ n'est autre que l'ensemble des distributions dont la dérivée appartient à $B V(\mathbb{R})$. On munit $B H(\mathbb{R})$ de la semi-norme

$$
\|f\|_{B H}:=\nu\left(f^{\prime}\right)+\left\|f^{\prime}\right\|_{\infty} .
$$


Les propositions 5 et 7 ont la conséquence immédiate que voici :

Proposition 8 1) On a $\dot{\mathcal{B}}_{1}^{2,1}(\mathbb{R}) \subset B H(\mathbb{R})$ et $\|f\|_{B H} \leq c\|f\|_{\dot{B}_{1}^{2,1}(\mathbb{R})}$, pour tout $f \in \dot{\mathcal{B}}_{1}^{2,1}(\mathbb{R})$.

2) Pour tout $f \in B H(\mathbb{R})$, on $a[f] \in \dot{B}_{1}^{2, \infty}(\mathbb{R})$ et $\|f\|_{\dot{B}_{1}^{2, \infty}(\mathbb{R})} \leq c\|f\|_{B H}$.

L'espace $B H(\mathbb{R})$ possède une remarquable propriété de stabilité par composition, établie par Savaré et Tomarelli [25] (voir aussi [13, th. 3] pour une preuve alternative).

Théorème 2 Si $f$ et $g$ appartiennent à $B H(\mathbb{R})$, il en est de même pour $f \circ g$ et l'on $a$

$$
\|f \circ g\|_{B H} \leq c\|f\|_{B H}\|g\|_{B H}
$$

\subsection{Modules de continuité}

Nous avons privilégié l'approche des espaces de Besov via la théorie de Littlewood-Paley. Ce point de vue, initié par Peetre [22] et Triebel [26], fait aujourd'hui référence en analyse non linéaire, comme en témoignent les travaux de Bony [2], Chemin [15] et de bien d'autres. Cependant la définition originale de Besov [1], à l'aide des modules de continuité, reste fort utile. Introduisons les opérateurs de différence finie

$$
\Delta_{h} f:=\tau_{-h} f-f \quad, \forall h \in \mathbb{R}^{n},
$$

et notons $\left(e_{1}, \ldots, e_{n}\right)$ la base canonique de $\mathbb{R}^{n}$. On dispose alors de la caractérisation suivante :

Proposition 9 Soient $s>0, p, q \in[1,+\infty]$ et un entier $m>s$. Alors tout élément de $\dot{B}_{p}^{s, q}\left(\mathbb{R}^{n}\right)$ possède un représentant $f \in L_{l o c}^{p}\left(\mathbb{R}^{n}\right)$ tel que

$$
\sum_{j=1}^{n}\left(\int_{\mathbb{R}}\left(\frac{1}{|h|^{s}}\left(\int_{\mathbb{R}^{n}}\left|\Delta_{h e_{j}}^{m} f(x)\right|^{p} d x\right)^{1 / p}\right)^{q} \frac{d h}{|h|}\right)^{1 / q}<+\infty
$$

et l'expression (2.9) est une norme équivalente à la norme $\|-\|_{\dot{B}_{p}^{s, q}\left(\mathbb{R}^{n}\right)}$ sur l'espace réalisé $\sigma_{m-1}\left(\dot{B}_{p}^{s, q}\left(\mathbb{R}^{n}\right)\right)$.

L'homologue de la proposition 9 pour les espaces de Besov non homogènes $B_{p}^{s, q}\left(\mathbb{R}^{n}\right)$ est bien connue (cf. par exemple Triebel [27, th. 2.6.1]). Par contre il n'y a pas de référence complète dans le cas homogène, peut-être en raison d'une forme de tabou frappant ces espaces fonctionnels. Disons que la preuve apparaît pour l'essentiel dans la monographie de Peetre [22], au chapitre 8 (voir aussi les notes du premier auteur [5]). 


\section{Le calcul fonctionnel}

\subsection{Rappel du cas inhomogène}

Le calcul fonctionnel sous-linéaire dans les espaces $B_{p}^{s, q}\left(\mathbb{R}^{n}\right)$ est l'objet du théorème suivant de Lanza, Sickel et du premier auteur [13, th. 11].

Théorème 3 Soient $1 \leq p<+\infty, q \in[1,+\infty]$ et $0<s<1+(1 / p)$. Si $f \in U_{p}^{1}(\mathbb{R})$ et $f(0)=0$, alors $T_{f}$ envoie $B_{p}^{1+(1 / p), 1}\left(\mathbb{R}^{n}\right)$ dans $B_{p}^{1+(1 / p), \infty}\left(\mathbb{R}^{n}\right)$ et $B_{p}^{s, q}\left(\mathbb{R}^{n}\right)$ dans lui-même. De plus il existe des constantes $c>0$ telles que

$$
\begin{aligned}
\|f \circ g\|_{B_{p}^{1+(1 / p), \infty}\left(\mathbb{R}^{n}\right)} & \leq c\|f\|_{U_{p}^{1}}\|g\|_{B_{p}^{1+(1 / p), 1}\left(\mathbb{R}^{n}\right)}, \quad \forall g \in B_{p}^{1+(1 / p), 1}\left(\mathbb{R}^{n}\right) \\
\|f \circ g\|_{B_{p}^{s, q}\left(\mathbb{R}^{n}\right)} & \leq c\|f\|_{U_{p}^{1}}\|g\|_{B_{p}^{s, q}\left(\mathbb{R}^{n}\right)}, \quad \forall g \in B_{p}^{s, q}\left(\mathbb{R}^{n}\right) .
\end{aligned}
$$

Preuve. En fait seul le cas $p>1$ est énoncé par les trois auteurs précités. Voici donc la preuve du théorème 3 dans le cas $p=1$.

Soit $f \in B H(\mathbb{R})$. En dimension 1, la proposition 8 et le théorème 2 conduisent à l'estimation

$$
\|f \circ g\|_{\dot{B}_{1}^{2, \infty}(\mathbb{R})} \leq c\|f\|_{B H}\|g\|_{\dot{B}_{1}^{2,1}(\mathbb{R})},
$$

pour tout $g \in B_{1}^{2,1}(\mathbb{R})$, alors que la condition $f(0)=0$ nous donne

$$
\|f \circ g\|_{1} \leq\left\|f^{\prime}\right\|_{\infty}\|g\|_{1} .
$$

En combinant les estimations (3.3) et (3.4), on obtient (3.1) dans le cas $n=p=1$. L'extension aux dimensions supérieures se fait exactement comme dans le cas $p>1$ (voir la preuve du théorème 11 de [13]). Il suffit de remplacer les différences secondes par des différences d'ordre 3, conformément à la proposition 9. L'estimation (3.2) se déduit de l'estimation (3.1) par interpolation non linéaire, y compris pour $p=1$.

\section{2. Énoncé et démonstration du théorème principal}

Théorème 4 Soient $1 \leq p<\infty, q \in[1,+\infty], 0<s<1+(1 / p)$ et $f \in U_{p}^{1}(\mathbb{R})$.

(i) Pour tout $g \in \dot{\mathcal{B}}_{p}^{1+(1 / p), 1}\left(\mathbb{R}^{n}\right)$, on a $[f \circ g] \in \dot{B}_{p}^{1+(1 / p), \infty}\left(\mathbb{R}^{n}\right), \partial_{j}(f \circ g) \in$ $\widetilde{C}_{0}\left(\mathbb{R}^{n}\right)$, pour tout $j=1, \ldots, n$, et

$$
\|f \circ g\|_{\dot{B}_{p}^{1+(1 / p), \infty}\left(\mathbb{R}^{n}\right)} \leq c\|f\|_{U_{p}^{1}}\|g\|_{\dot{B}_{p}^{1+(1 / p), 1}\left(\mathbb{R}^{n}\right)} .
$$

(ii) Pour tout $g \in \dot{\mathcal{B}}_{p}^{s, q}\left(\mathbb{R}^{n}\right)$, on a $f \circ g \in \dot{\mathcal{B}}_{p}^{s, q}\left(\mathbb{R}^{n}\right)$ et

$$
\|f \circ g\|_{\dot{B}_{p}^{s, q}\left(\mathbb{R}^{n}\right)} \leq c\|f\|_{U_{p}^{1}}\|g\|_{\dot{B}_{p}^{s, q}\left(\mathbb{R}^{n}\right)} .
$$


Preuve. Soit $f$ une fonction de la classe $U_{p}^{1}(\mathbb{R})$. En retranchant à $f$ une constante, on peut supposer, sans perte de généralité, que $f(0)=0$.

Supposons d'abord que $g \in B_{p}^{s, q}\left(\mathbb{R}^{n}\right)$. Posons $g_{\lambda}(x):=g(\lambda x)$, pour tous $\lambda>0$ et $x \in \mathbb{R}^{n}$. En renormant au besoin $\dot{B}_{p}^{s, q}\left(\mathbb{R}^{n}\right)$ (voir la relation $(2.5)$ ), on a

$$
\left\|g_{\lambda}\right\|_{B_{p}^{s, q}\left(\mathbb{R}^{n}\right)}=\lambda^{s-(n / p)}\|g\|_{\dot{B}_{p}^{s, q}\left(\mathbb{R}^{n}\right)}+\lambda^{-n / p}\|g\|_{p} .
$$

En appliquant le théorème 3 , et en écrivant l'identité (3.7) pour la fonction $f \circ g_{\lambda}=(f \circ g)_{\lambda}$, on obtient

$$
\begin{aligned}
& \lambda^{s-(n / p)}\|f \circ g\|_{\dot{B}_{p}^{s, q}\left(\mathbb{R}^{n}\right)}+\lambda^{-n / p}\|f \circ g\|_{p} \\
& \quad \leq c\|f\|_{U_{p}^{1}}\left(\lambda^{s-(n / p)}\|g\|_{\dot{B}_{p}^{s, q}\left(\mathbb{R}^{n}\right)}+\lambda^{-n / p}\|g\|_{p}\right) .
\end{aligned}
$$

En divisant alors par $\lambda^{s-(n / p)}$ et en faisant tendre $\lambda$ vers $+\infty$, on obtient précisément l'estimation (3.6). Le même argument d'échelle conduit à l'estimation (3.5).

Venons-en au cas général où $g \in \dot{\mathcal{B}}_{p}^{s, q}\left(\mathbb{R}^{n}\right)$. D'après la proposition 6 , on dispose d'une suite $\left(g_{k}\right)_{k \geq 1}$ dans $B_{p}^{s, q}\left(\mathbb{R}^{n}\right)$ telle que

$$
\left\|g_{k}\right\|_{\dot{B}_{p}^{s, q}\left(\mathbb{R}^{n}\right)} \leq c\|g\|_{\dot{B}_{p}^{s, q}\left(\mathbb{R}^{n}\right)} \quad, \forall k \geq 1
$$

et d'une suite numérique $\left(a_{k}\right)_{k \geq 1}$ telle que $g_{k}+a_{k}$ tende vers $g$ dans $L_{l o c}^{1}\left(\mathbb{R}^{n}\right)$. En appliquant le résultat de la première étape aux fonctions $\tau_{-a_{k}} f$ et en notant que $U_{p}^{1}(\mathbb{R})$ est isométriquement invariant par translation, on voit que

$$
\left\|f \circ\left(g_{k}+a_{k}\right)\right\|_{\dot{B}_{p}^{s, q}\left(\mathbb{R}^{n}\right)} \leq c\|f\|_{U_{p}^{1}}\|g\|_{\dot{B}_{p}^{s, q}\left(\mathbb{R}^{n}\right)} .
$$

Sachant que $f$ est lipschitzienne, on vérifie aussitôt que la suite

$$
\left(f \circ\left(g_{k}+a_{k}\right)\right)_{k \geq 1}
$$

tend vers $f \circ g$ dans $L_{l o c}^{1}\left(\mathbb{R}^{n}\right)$. Dès lors on peut appliquer la proposition 2 et conclure que les estimations (3.5) et (3.6) sont vérifiées en toute généralité.

Nous terminerons la preuve du théorème en établissant que

$$
\partial_{j}(f \circ g) \in \widetilde{C}_{0}\left(\mathbb{R}^{n}\right) \quad \text {, pour } j=1, \ldots, n .
$$

On utilise à nouveau la preuve de la proposition 5 .

Dans le cas $s<n / p$ (ou $s=n / p$ et $q=1$ ), on a $g=h_{1}+h_{2}$, où $h_{1}$ est une fonction continue ayant une limite finie à l'infini, et $h_{2} \in L^{p}\left(\mathbb{R}^{n}\right)$. La fonction $f \circ h_{1}$ est alors continue et bornée. On a de plus

$$
\int_{\mathbb{R}^{n}}\left|f(g(x))-f\left(h_{1}(x)\right)\right|^{p} d x \leq\left\|f^{\prime}\right\|_{\infty}^{p}\left\|h_{2}\right\|_{p}^{p}
$$

et donc $f \circ g-f \circ h_{1} \in L^{p}\left(\mathbb{R}^{n}\right)$. Ainsi la propriété (3.8) est satisfaite. 
Si $n / p \leq s<(n / p)+1$, on a $g=h_{1}+h_{2}$, où $h_{1} \in E\left(\mathbb{R}^{n}\right)$ et $h_{2} \in L^{p}\left(\mathbb{R}^{n}\right)$. Puisque $f$ est lipschitzienne, la fonction $f \circ h_{1}$ appartient aussi à $E\left(\mathbb{R}^{n}\right)$. La propriété (3.8) découle alors du lemme 1.

Enfin pour $s=1+(n / p)$ et $n=q=1$, on a $g^{\prime} \in C_{0}(\mathbb{R})$. Puisque $(f \circ g)^{\prime}=\left(f^{\prime} \circ g\right) g^{\prime}$ au sens des distributions, on en déduit aisément que $(f \circ g)^{\prime} \in \widetilde{C}_{0}(\mathbb{R})$.

\subsection{Remarques}

1.- Signalons une preuve alternative du théorème 4 . Elle consiste à traiter d'abord le cas limite $s=1+(1 / p)$, en adaptant aux espaces homogènes la preuve du théorème 11 de [13], puis à utiliser un argument d'interpolation non linéaire. Cette approche a l'avantage d'aborder directement le cas homogène, mais elle est plus longue et techniquement plus délicate.

2.- Nous avons établi le théorème 4 pour $0<s \leq 1+(1 / p)$, alors que seul l'intervalle $1 \leq s \leq 1+(1 / p)$ justifiait nos efforts. En effet, pour $0<s<1$, la proposition 9 entraîne aussitôt que la fonction $f$ opère sur $\dot{B}_{p}^{s, q}\left(\mathbb{R}^{n}\right)$ (ou, plus exactement, sur $\left.\sigma_{0}\left(\dot{B}_{p}^{s, q}\left(\mathbb{R}^{n}\right)\right)\right)$, sous la seule condition de Lipschitz.

3.- Que deviennent nos résultats dans le cas $p=\infty$ ? On vient de rappeler ce qu'il advient pour $0<s<1$. Concernant le cas limite $s=1$, la condition de Lipschitz suffit pour que l'opérateur $T_{f}$ envoie $\dot{B}_{\infty}^{1,1}\left(\mathbb{R}^{n}\right)$ dans $\dot{B}_{\infty}^{1, \infty}\left(\mathbb{R}^{n}\right)$; ce résultat découle aisément d'un argument de Lanza et du premier auteur [12, prop. 4].

\section{Une application}

L'espace fonctionnel $B V\left(\mathbb{R}^{2}\right)$ joue un rôle central dans le modèle d'OsherRudin pour la compression d'image (cf. Meyer [19]). Cependant il n'admet pas de description élémentaire au moyen des coefficients d'ondelettes. Il en va tout autrement pour son proche parent, l'espace de Besov $\dot{B}_{1}^{1, \infty}\left(\mathbb{R}^{2}\right)$, pour lequel on dispose de l'équivalence de normes

$$
\left\|\sum_{\varepsilon=1}^{3} \sum_{j \in \mathbb{Z}} \sum_{k \in \mathbb{Z}^{2}} c_{\varepsilon, j, k} 2^{j} \psi_{\varepsilon}\left(2^{j}(.)-k\right)\right\| \sim \sum_{\varepsilon=1}^{3} \sup _{j \in \mathbb{Z}}\left(\sum_{k \in \mathbb{Z}^{2}}\left|c_{\varepsilon, j, k}\right|\right),
$$

dès que $\left(\psi_{1}, \psi_{2}, \psi_{3}\right)$ est un système d'ondelettes suffisamment régulières qui engendre une base hilbertienne de $L^{2}\left(\mathbb{R}^{2}\right)$ (voir [8]). Nous nous proposons d'établir qu'une fonction étagée appartient à $B V\left(\mathbb{R}^{2}\right)$ si et seulement si elle appartient à $\dot{B}_{1}^{1, \infty}\left(\mathbb{R}^{2}\right)$. La comparaison des normes d'une telle fonction, dans les deux espaces fonctionnels, est un problème plus délicat, auquel nous apporterons une réponse partielle, vraisemblablement perfectible. 
Jusqu'à la fin de l'article, on remplacera la semi-norme $\|-\|_{\dot{B}_{1}^{1, \infty}\left(\mathbb{R}^{2}\right)}$ par la semi-norme équivalente mise en évidence en (2.9). Nous poserons donc

$$
\|f\|_{\dot{B}_{1}^{1, \infty}\left(\mathbb{R}^{2}\right)}:=\sup _{y \in \mathbb{R}^{2} \backslash\{0\}} \frac{1}{|y|} \int_{\mathbb{R}^{2}}|f(x+y)+f(x-y)-2 f(x)| d x .
$$

Commençons par énoncer le corollaire du théorème 4 qui nous sera utile.

Corollaire 1 Pour tout intervalle compact I, définissons la fonction $u_{I}$ par

$$
u_{I}(x):=\int_{-\infty}^{x} \mathbf{1}_{I}(t) d t \quad, x \in \mathbb{R} .
$$

Il existe une constante $c_{0}>0$ telle que

$$
\left\|u_{I} \circ f\right\|_{\dot{B}_{1}^{1, \infty}\left(\mathbb{R}^{n}\right)} \leq c_{0}\|f\|_{\dot{B}_{1}^{1, \infty}\left(\mathbb{R}^{n}\right)},
$$

quel que soit $f \in \dot{\mathcal{B}}_{1}^{1, \infty}\left(\mathbb{R}^{n}\right)$ et quel que soit I.

Preuve. Il suffit d'observer que $\left\|u_{I}\right\|_{B H}=\left\|\mathbf{1}_{I}^{\prime}\right\|_{M}+\left\|\mathbf{1}_{I}\right\|_{\infty}=3$.

Théorème 5 Soit $E$ un ensemble borélien arbitraire de $\mathbb{R}^{2}$ et $f$ la fonction indicatrice de $E$. Alors $f$ appartient à $B V\left(\mathbb{R}^{2}\right)$ si et seulement si elle appartient à $\dot{\mathcal{B}}_{1}^{1, \infty}\left(\mathbb{R}^{2}\right)$ et l'on a

$$
\frac{1}{2}\|f\|_{\dot{B}_{1}^{1, \infty}\left(\mathbb{R}^{2}\right)} \leq\|f\|_{B V} \leq\|f\|_{\dot{B}_{1}^{1, \infty}\left(\mathbb{R}^{2}\right)} .
$$

Preuve. Dans la proposition 7 , on a déjà obtenu l'inclusion $B V\left(\mathbb{R}^{2}\right) \subset$ $\dot{\mathcal{B}}_{1}^{1, \infty}\left(\mathbb{R}^{2}\right)$, ainsi que l'estimation $\|f\|_{\dot{B}_{1}^{1, \infty}\left(\mathbb{R}^{2}\right)} \leq c\|f\|_{B V}$. Avec le choix (4.1) pour la norme $\dot{B}_{1}^{1, \infty}$, on voit aussitôt qu'on peut prendre $c=2$. La partie subtile du théorème 5 est évidemment la seconde inégalité. Pour établir cette estimation, on utilise le lemme suivant.

Lemme 2 Si $a, b$ et c appartiennent tous trois $\grave{a}\{0,1\}$, alors

$$
|a-c| \leq|a+b-2 c|
$$

En effet, si $a+b-2 c \neq 0$, ceci est évident, car on a $|a-c| \leq 1 \leq|a+b-2 c|$. Si $a+b-2 c=0$, on a $c=\frac{a+b}{2}$ ce qui entraîne $a=b=c$, car tous trois appartiennent à $\{0,1\}$. Alors $|a-c|=0=|a+b-2 c|$.

On applique le lemme 2 à $a:=f(x+y), b:=f(x-y)$ et $c:=f(x)$, lorsque $f:=\mathbf{1}_{E}$, ce qui termine la preuve du théorème 5 . 
Théorème 6 Soit $f$ une fonction étagée sur $\mathbb{R}^{2}$ et soit $N$ le nombre de valeurs prises par $f$. Alors $f$ appartient à $B V\left(\mathbb{R}^{2}\right)$ si et seulement si elle appartient à $\dot{\mathcal{B}}_{1}^{1, \infty}\left(\mathbb{R}^{2}\right)$ et l'on a

$$
\frac{1}{2}\|f\|_{\dot{B}_{1}^{1, \infty}\left(\mathbb{R}^{2}\right)} \leq\|f\|_{B V} \leq c_{0}(N-1)\|f\|_{\dot{B}_{1}^{1, \infty}\left(\mathbb{R}^{2}\right)},
$$

où c est la constante du corollaire 1.

Preuve. Si $N=1, f$ est une constante, qui vérifie trivialement les inégalités (4.2).

Supposons $N>1$ et désignons par $a_{1}<a_{2}<\cdots<a_{N}$ la suite des valeurs de $f$. On pose

$$
\Omega_{k}:=\left\{x: f(x) \geq a_{k}\right\}
$$

pour $k=1, \ldots, N$. On a alors

$$
f=\sum_{k=1}^{N-1} a_{k} \mathbf{1}_{\Omega_{k} \backslash \Omega_{k+1}}+a_{N} \mathbf{1}_{\Omega_{N}}=a_{1}+\sum_{k=2}^{N}\left(a_{k}-a_{k-1}\right) \mathbf{1}_{\Omega_{k}} .
$$

Appliquons le corollaire 1 aux intervalles $I_{k}:=\left[a_{k-1}, a_{k}\right]$. Puisque

$$
\left(a_{k}-a_{k-1}\right) \mathbf{1}_{\Omega_{k}}=u_{I_{k}} \circ f \quad, \quad k=2, \ldots, N,
$$

il vient

$$
\left\|\left(a_{k}-a_{k-1}\right) \mathbf{1}_{\Omega_{k}}\right\|_{\dot{B}_{1}^{1, \infty}\left(\mathbb{R}^{2}\right)} \leq c_{0}\|f\|_{\dot{B}_{1}^{1, \infty}\left(\mathbb{R}^{2}\right)} .
$$

En combinant $(4.3),(4.4)$ et le théorème 5 , on obtient

$$
\|f\|_{B V} \leq c_{0}(N-1)\|f\|_{\dot{B}_{1}^{1, \infty}\left(\mathbb{R}^{2}\right)} .
$$

Nous allons voir qu'en nous restreignant à des fonctions étagées "raisonnables", on obtient l'équivalence (4.2) avec des constantes indépendantes $\mathrm{du}$ nombre de valeurs prises par la fonction. Supposons en effet que $f=$ $a_{1} \mathbf{1}_{\Omega_{1}}+\cdots+a_{N} \mathbf{1}_{\Omega_{N}}$, où les $\Omega_{k}$ sont des ouverts bornés tels que $\Omega_{N} \subset \cdots \subset \Omega_{1}$ et dont les frontières $\Gamma_{1}, \ldots, \Gamma_{N}$ sont deux à deux disjointes. Supposons en outre que $a_{j}>0$, pour $j=1, \ldots, N$. On a alors

$$
c_{1}\|f\|_{\dot{B}_{1}^{1, \infty}\left(\mathbb{R}^{2}\right)} \leq\|f\|_{B V} \leq c_{2}\|f\|_{\dot{B}_{1}^{1, \infty}\left(\mathbb{R}^{2}\right)},
$$

où les deux constantes $c_{2}>c_{1}>0$ ne dépendent pas de $N$, mais seulement de la définition des semi-normes $B V$ et $\dot{B}_{1}^{1, \infty}$.

Pour démontrer (4.5), on utilise la remarque suivante : 
Lemme 3 Il existe une constante $c>0$ telle que, pour tout $f \in B V\left(\mathbb{R}^{n}\right)$, on ait

$$
\|f\|_{B V} \leq c \limsup _{y \rightarrow 0} \frac{1}{|y|} \int_{\mathbb{R}^{n}}|f(x+y)-f(x)| d x .
$$

Pour démontrer (4.6), on désigne par $\lambda$ la limite sup qui apparaît au second membre. Pour tout $\varepsilon>0$, il existe donc $\eta(\varepsilon)>0$ tel que

$$
\int_{\mathbb{R}^{n}}|f(x+y)-f(x)| d x \leq|y|(\lambda+\varepsilon), \quad \text { si }|y| \leq \eta(\varepsilon) .
$$

On teste $(4.7)$ si $y=r e_{j}, j=1, \ldots, n$, où $0<r \leq \eta(\varepsilon)$. Alors (4.7) implique

$$
\left\|\partial_{j} f\right\|_{M} \leq \lambda+\varepsilon \quad, \quad j=1, \ldots, n .
$$

Puisque (4.8) a lieu pour tout $\varepsilon>0$, on obtient (4.6).

On écrit

$$
f(x+y)+f(x-y)-2 f(x)=\sum_{j=1}^{N} a_{j}\left(\chi_{j}(x+y)+\chi_{j}(x-y)-2 \chi_{j}(x)\right),
$$

où, pour simplifier les notations, on a posé $\chi_{j}:=\mathbf{1}_{\Omega_{j}}$. Le support de la fonction

$$
x \mapsto \chi_{j}(x+y)+\chi_{j}(x-y)-2 \chi_{j}(x)
$$

est inclus dans $\widetilde{\Gamma}_{j}:=\left\{x: \operatorname{dist}\left(x, \Gamma_{j}\right) \leq|y|\right\}$. Si $|y|$ est suffisamment petit, les ensembles $\widetilde{\Gamma}_{j}$ sont deux à deux disjoints (car les $\Gamma_{j}$ le sont et sont compacts); on a donc

$$
\begin{aligned}
\int_{\mathbb{R}^{2}} \mid f(x+y)+f & (x-y)-2 f(x) \mid d x \\
= & \sum_{j=1}^{N} a_{j} \int_{\mathbb{R}^{2}}\left|\chi_{j}(x+y)+\chi_{j}(x-y)-2 \chi_{j}(x)\right| d x
\end{aligned}
$$

On utilise encore le lemme 2 . Il vient

$$
\left|\chi_{j}(x+y)+\chi_{j}(x-y)-2 \chi_{j}(x)\right| \geq\left|\chi_{j}(x+y)-\chi_{j}(x)\right|
$$

et donc

$$
\int_{\mathbb{R}^{2}}|f(x+y)+f(x-y)-2 f(x)| d x \geq \int_{\mathbb{R}^{2}}|f(x+y)-f(x)| d x,
$$

si $|y|$ est suffisamment petit. Il suffit alors d'utiliser (4.6) pour conclure. 


\section{Références}

[1] Besov, O. V. : Investigation of a class of function spaces in connection with imbedding and extension theorems. (Russian) Trudy. Mat. Inst. Steklov 60 (1961), 42-81.

[2] Bony, J. M. : Calcul symbolique et propagation des singularités pour les équations aux dérivées partielles non linéaires. Ann. Sci. École Norm. Sup. (4) 14 (1981), 209-246.

[3] Bourdaud, G. : Réalisations des espaces de Besov homogènes. Ark. Mat. 26 (1988), 41-54.

[4] Bourdaud, G. : Fonctions qui opèrent sur les espaces de Besov et de Triebel. Ann. Inst. H. Poincaré Anal. Non Linéaire 10 (1993), 413-422.

[5] Bourdaud, G. : Ce qu'il faut savoir sur les espaces de Besov. Prépub. Univ. Paris 7 - URA 212, 53 (1993).

[6] Bourdaud, G. : The functional calculus in Sobolev spaces. In Function spaces, differential operators and non linear analysis (Friedrichroda, 1992), 127-142. Teubner-Texte Math. 133. Teubner, Stuttgart, 1993.

[7] Bourdaud, G. : Calcul fonctionnel dans certains espaces de LizorkinTriebel. Arch. Math. (Basel) 64 (1995), 42-47.

[8] Bourdaud, G. : Ondelettes et espaces de Besov. Rev. Mat. Iberoamericana 11 (1995), no. 3, 477-512.

[9] Bourdaud, G. : Superposition operators in Zygmund and BMO spaces. In Function spaces, differential operators and non linear analysis (Teistungen, 2001), 59-74. Birkhäuser, Basel, 2003.

[10] Bourdaud, G. and Kateb, M. E. D. : Calcul fonctionnel dans l'espace de Sobolev fractionnaire. Math. Z. 210 (1992), 607-613.

[11] Bourdaud, G. And Kateb, M. E. D. : Fonctions qui opèrent sur les espaces de Besov. Math. Ann. 303 (1995), 653-675.

[12] Bourdaud, G. and Lanza de Cristoforis, M. : Functional calculus in Hölder-Zygmund spaces. Trans. Amer. Math. Soc. 354 (2002), 4109-4129.

[13] Bourdaud, G., Lanza de Cristoforis, M. and Sickel, W. : Superposition operators and functions of bounded $p$-variation. Rev. Mat. Iberoamericana, 22 (2006), no. 2, 455-487.

[14] Bourdaud, G. ANd Meyer, Y. : Fonctions qui opèrent sur les espaces de Sobolev. J. Funct. Anal. 97 (1991), 351-360.

[15] Chemin, J.-Y. : Fluides parfaits incompressibles. Astérisque 230 (1995).

[16] DeVore, R. A. And Lorentz, G. G. : Constructive approximation. Fundamental Principles of Mathematical Sciences 303. Springer, Berlin, 1993.

[17] Franke, J. : On the space $F_{p q}^{s}$ of Triebel-Lizorkin type : pointwise multipliers and spaces on domains. Math. Nachr. 125 (1986), 29-68.

[18] Lemarié-Rieusset, P. G. : Recent developments in the Navier-Stokes problem. Chapman Hall/CRC Research Notes in Mathematics 431. Chapman Hall/CRC, Boca Raton, FL, 2002. 
[19] Meyer, Y. : Oscillating patterns in image processing and nonlinear evolution equations. The fifteenth Dean Jacqueline B. Lewis memorial lectures. University Lecture Series, 22, Amer. Math. Soc., Providence, RI, 2001.

[20] Oswald, P. : On the boundedness of the mapping $f \rightarrow|f|$ in Besov spaces. Comment. Math. Univ. Carolin. 33 (1992), 57-66.

[21] Peetre, J. : Interpolation of Lipschitz operators and metric spaces. Mathematica (Cluj) 12 (1970), 325-334.

[22] Peetre, J. : New thoughts on Besov spaces. Duke Univ. Math. Series I. Mathematics Department, Duke University, Durham, N.C., 1976.

[23] Runst, T. And Sickel, W. : Sobolev spaces of fractional order, Nemytskij operators, and nonlinear partial differential equations. De Gruyter Series in Nonlinear Analysis and Applications 3. Walter de Gruyter, Berlin, 1996.

[24] Savaré, G. : On the regularity of the positive part of functions. Nonlinear Anal. 27 (1996), no. 9, 1055-1074.

[25] Savaré, G. and Tomarelli, F. : Superposition and chain rule for bounded Hessian functions. Adv. Math. 140 (1998), 237-281.

[26] TRIeBel, H. : Interpolation theory, function spaces, differential operators. North-Holland Mathematical Library 18. North-Holland, Amsterdam-New York, 1978.

[27] Triebel, H. : Theory of function spaces II. Monographs in Mathematics 84. Birkhäuser, Basel, 1992.

Recibido: 11 de noviembre de 2004

Gérard Bourdaud

Institut de Mathématiques de Jussieu Projet d'analyse fonctionnelle, Case 186

4 place Jussieu, 75252

Paris Cedex 05, France bourdaud@ccr.jussieu.fr

Yves Meyer

CMLA, ENS-Cachan

94235 Cachan Cedex, France ymeyer@cmla.ens-cachan.fr 